\title{
Asymmetric vector mesons produced in nuclear collisions
}

\author{
I.M. Dremin, V.A. Nechitailo \\ Lebedev Physical Institute, Moscow 119991, Russia \\ National Research Nuclear University "MEPhI", Moscow 115409, Russia
}

\begin{abstract}
It is argued that the experimentally observed phenomenon of asymmetric shapes of vector mesons produced in nuclear media during high energy nucleus-nucleus collisions can be explained as Fano-Feshbach resonances. It has been observed that the mass distributions of lepton pairs created at meson decays decline from the traditional BreitWigner shape with some excess in the low-mass wing of the resonance. It is clear that the whole phenomenon is related to some interaction with the nuclear medium. Moreover, it can be further detalized in quantum mechanics as the interference of direct and continuum states in Fano-Feshbach effect. To reveal the nature of the interaction it is proposed to use a phenomenological model of the additional contribution due to Cherenkov gluons. They can be created because of the excess of the refractivity index over 1 just in the low-mass wing as required by the classical Cherenkov treatment. In quantum mechanics, this requirement is related to the positive real part of the interaction amplitude in this wing. The corresponding parameters are found from the comparison with $\rho$-meson data and admit reasonable explanation.
\end{abstract}

\section{Introduction}

Resonance peaks are observed in many natural phenomena. They are treated in numerous textbooks (see, e.g., [1]). The traditional way of their description is to compare their shapes with the relativistic Breit-Wigner formula [2, 3]

$$
f=\frac{k}{\left(m_{r}^{2}-M^{2}\right)^{2}+M^{2} \Gamma_{r}^{2}},
$$

where $f$ denotes a signal strength, $k$ is the normalization constant, $M$ is the scanning energy, $m_{r}$ is the maximum position (the resonance mass), $\Gamma_{r}$ 
is the resonance width. One measures the signal intensity $f$ at different energies $M$. For example, the atoms considered as oscillators emit as BreitWigner resonances. The resonance shape is a purely statistical phenomenon. It depends on many details of interactions and need not to be necessarily symmetric.

The asymmetric resonance peaks were experimentally observed in various fields of physics even before the formula (1) was proposed. E.g., the resonance of He atoms observed in the inelastic scattering of electrons is strongly asymmetric (see [4, 5]). Many spectroscopic studies of atoms were devoted to this phenomenon. The name of Fano resonances was attached to observed asymmetric resonances. In nuclear physics, they are known as Feshbach resonances [6].

Both types of resonances (we can call them as FF-resonances) are the same thing when it comes to their mathematical essence [5, 7, 6]. The asymmetry is explained as a byproduct of the quantum-mechanical interference between two separate channels of the reaction. Namely, interference between a background (continuum of states) and a resonant (excitation of the discrete states) scattering process produces the asymmetric line-shapes. The two separate channels (closed and open) differ but they couple to each other. The resonance has an energy width that depends on the coupling between the channels.

In particle physics, such peaks are identified with unstable particles. Let us mention, however, that sometimes even the "ordinary" hadrons are treated as FF-resonances [8]. It is usually claimed that the symmetric shape is observed for resonances directly produced in particle collisions. Their characteristics are compiled by the PDG (Particle Data Group) [3]. However, some asymmetry was recently experimentally noticed for narrow resonances (with c- and b-quarks). It was explained as a consequence of FF-effects [9, 10] with interference of bound states and continuum induced by vacuum excitation of light quark-antiquark pairs leading to creation of D-mesons.

The situation has strongly changed after the high energy nucleus-nucleus collisions became available. The created particles have to leak somehow from the nuclear medium. Medium interactions may lead to some modification of their characteristics. Really, there are numerous experimental data [11, 12, 13, 14, 15, 16, 17, 18, 19, 20, about the in-medium modification of widths and positions of prominent wide vector meson resonances. Some of them even contradict each other. They are mainly obtained from the shapes of dilepton (decay products) mass and transverse momentum spectra in nucleus- 
nucleus collisions. Some excess over the expected symmetric shape of the distribution was observed. Dilepton spectroscopy directly probes the vector component of the spectral function of the hadronic medium. The dilepton mass spectra decrease approximately exponentially with increase of masses but show peaks over this trend at some masses which can be identified with prominent resonances. The $\rho$-meson peak is usually the strongest one [11, 12, 13, 14] in the ratio $\rho: \omega: \phi=10: 1: 2$. Below, we concentrate on properties of in-medium $\rho$-mesons with the special attention to be paid to the asymmetry of their shape.

Several approaches have been advocated for explanation of the observed excess and properties of in-medium resonances [21, 22, 23, 24, 25, 26, 27, 28, 29, 30, 31, 32, 33. Most of them use effective hadronic Lagrangians to compute loop corrections and/or just hydrodynamics ideas of the expanding fireball. See also the review [34] on cold nuclear matter effects. However, either positions, widths or heights presented some problems. Therefore we will not review these attempts in detail. Here we concentrate not as much on their particular values which we just fit by the corresponding parameters as on the asymmetry of the resonance shape.

\section{Experiment, Fano-Feshbach-effect and Cherenkov gluons}

The dilepton mass spectrum in semi-central In-In collisions at 158 AGeV measured by NA60-Collaboration [13] is shown in Fig.1 by dots with error bars in the region of $\rho$ and $\phi, \omega$-mesons. Its asymmetry is easily seen with some excess in the low-mass wing. The shape is quite distinct from the familiar $\rho$-peak with PDG-parameters $m_{\rho}=775 \mathrm{MeV}, \Gamma_{\rho}=149 \mathrm{MeV}$ [3] shown in Fig. 1 by the dashed line.

The in-medium modification of $\rho$-meson parameters can not be accounted by simple variation of them within the Breit-Wigner formula (1). It is demonstrated by the dash-dotted line in Fig. 1 with fit parameters $m_{r}=775 \mathrm{MeV}$, $\Gamma_{r}=336 \mathrm{MeV}$ (much larger width!) which does not reproduce the observed asymmetry.

As described above, the interference of the continuum states and discrete levels of the reaction leads in quantum mechanics to the well known FFeffect [5, 7, 6]. They interfere with opposite phase on the two sides of the 


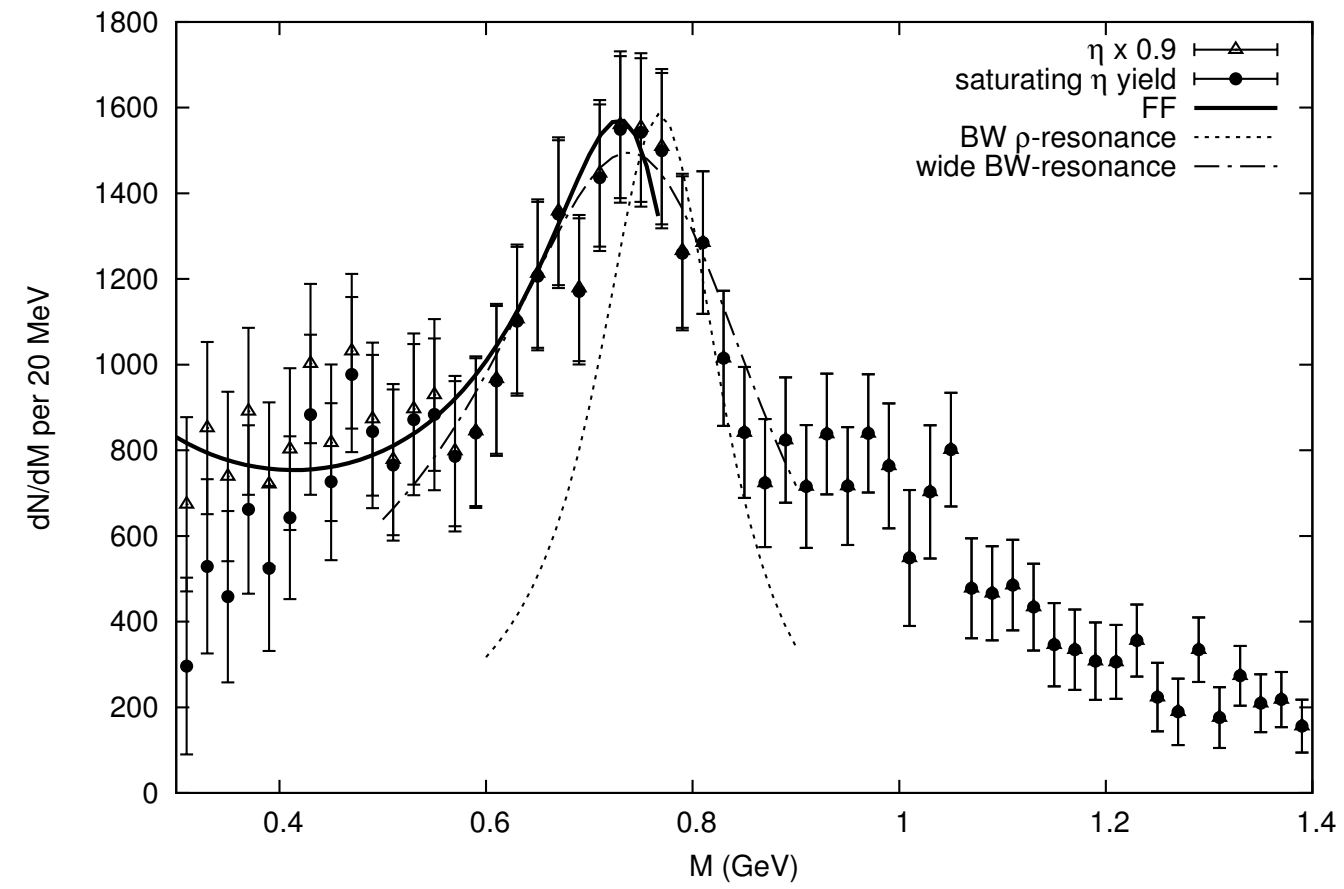

Figure 1: The spectrum of dileptons in semi-central collisions $\operatorname{In}(158 \mathrm{~A} \mathrm{GeV})$ In measured by NA60-Collaboration [14] (points with error bars). The solid line shows that the fits by Eq. (4) with FF-effect and by Eq. (6) with gluon Cherenkov effect taken into account coincide. The dashed line corresponds to the Breit-Wigner shape of $\rho$-meson with PDG-parameters [3]. The dashdotted line shows this shape with the modified width.

resonance as shown in [7]. The resonance asymmetry is described by the following formula derived in Ref. [7]:

$$
\sigma=\frac{(q+\epsilon)^{2}}{1+\epsilon^{2}}=1+\frac{q^{2}-1+2 q \epsilon}{\epsilon^{2}+1},
$$

where at the relativistic notation

$$
\epsilon=\frac{M^{2}-m_{r}^{2}}{M \Gamma_{r}} .
$$

This expression replaces its non-relativistic form in the original paper [5] to reproduce properly the contribution due to the Breit-Wigner resonance shape 
(1). The parameter $q$ describes the relative strength of discrete states and unperturbed continuum. The term linear in $\epsilon$ is in charge of asymmetry. After subtracting the constant background and normalizing the Breit - Wigner part, one gets the following expression which can be fitted to experimental data of Fig. 1 when multiplied by the overall normalization factor $C$ :

$$
C \frac{1-\sigma}{1-q^{2}}=C \frac{1-2 q \epsilon /\left(1-q^{2}\right)}{\epsilon^{2}+1} .
$$

Unfortunately, one can not use for the fitting procedure the experimental data in the whole region of masses $M$ shown in Fig. 1 because the admixture of higher mass resonances is large in the right wing of the Figure. Therefore we had to use the data in the smaller interval of masses below the peak, i.e., in the left wing only. The fit shown by the solid line in Fig. 1 resulted in the value of $q \approx 0.363\left(\Gamma_{r}=184 \mathrm{MeV}, C=29\right)$. Let us note that the width is larger than the ordinary one.

One concludes that the interference parameter $q$ is quite noticeable to explain the shape of the resonance with excess of mesons in the left wing (smaller masses) of the resonance profile.

The admixture of the contribution of direct states to this effect compared to the influence of the continuum was estimated in [7] equal $\pi q^{2} / 2$ which amounts to about 0.21 in our case. Thus we conclude that the interference of the continuum with quasibound states is quite important.

The above treatment is based on general quantum-mechanical principles and does not reveal what particular mechanisms are in charge of the interfering open and closed channels. One can propose emission of Cherenkov gluons in the nuclear media [35] as one of these channels that explains a possible source of the left-wing asymmetry.

The necessary condition for Cherenkov effects to be observable within some energy interval is an excess of the refractivity index of the medium $n$ over 1. It is well known for ordinary media that such an excess happens due to electromagnetic interactions just in the left wing of any resonance (e.g., see Fig. 31.5 in [1]). According to general formulas (e.g., see [36]) this excess is proportional to the real part of the forward (depicted by 0 below) scattering amplitude.

$$
\Delta n=\operatorname{Re} n-1 \propto \operatorname{Re} F(M, 0)>0 .
$$

For the nuclear quark-gluon medium this requirement should be fulfilled for the chromopermittivity of gluons [37]. The real part of the Breit-Wigner 
amplitude leading to Eq. (1) is positive just within the low-energy (left) wing of any resonance described by this equation (see, e.g., [29, 30]). Herefrom one gets the general prediction that the shape of any resonance formed in high energy nuclei collisions must become asymmetric with some excess within its left wing compared to the usual Breit-Wigner shape. One could expect that some collective excitations of the quark-gluon medium may contribute in these energy intervals in addition to the traditional effects. Since the probability of Cherenkov radiation is proportional to $\Delta n$ the asymmetry must be proportional to it. Then the dilepton mass distribution must get the shape (the formula in [35] is slightly corrected):

$$
\frac{d N_{l l}}{d M}=\frac{A}{\left(m_{r}^{2}-M^{2}\right)^{2}+M^{2} \Gamma_{r}^{2}}\left(1+w_{r} \frac{m_{r}^{2}-M^{2}}{M \Gamma_{r}} \Theta\left(m_{r}-M\right)\right) .
$$

This formula was also used to fit experimental data in Fig. 1. The second term is due to the coherent Cherenkov gluon response of the medium to the penetrating quark proportional to the real part of the amplitude. It is in charge of the observed asymmetry. It vanishes at energies above the resonance peak $M>m_{r}$ because only positive $\Delta n$ lead to Cherenkov effects. Here, we take into account that the ratio of real to imaginary parts of BreitWigner amplitudes is

$$
\frac{\operatorname{Re} F(M, 0)}{\operatorname{Im} F(M, 0)}=\frac{m_{r}^{2}-M^{2}}{M \Gamma_{r}} .
$$

The weight of the second term is described by the only adjustable parameter $w_{r}$ for a given resonance $r$. As we see, the general structure of Eqs (4), (6) is the same with

$$
w_{r}=2 q /\left(1-q^{2}\right) .
$$

The adjusted parameters were obtained from the independent fit to experimental points in Fig. 1. They are $A=25, \Gamma_{r}=184 \mathrm{MeV}, w_{r}=0.838$. The relation (8) is well fulfilled. Therefore both fits according to (4) and (6) practically coincide and are shown in Fig. 1 by a single solid line.

The quantum interference of continuum and quasibound states is at the origin both of asymmetric resonances and of the classical phenomenological prescription of $\Delta n>0$, which is required for Cherenkov effect. Therefore, FF-effect can serve as the quantum-mechanics foundation of classical Cherenkov effect in general. The overlap of both fits demonstrated by the solid line in Fig. 1 supports this conclusion. 


\section{QCD perspective}

At that stage one is tempted to speculate about the QCD interpretation of such a statement. High energy nuclei collisions give rise to a state of the boiling quark-gluon matter (plasma?). The numerous quark-antiquark pairs with different colors and masses (energy in the center of mass system of the pair) are produced there. The color-neutral pairs whose mass fits the Breit-Wigner shape form the resonance.

However, most pairs in the plasma are in a color-octet state and may not create resonances. They are considered as a continuum. After interaction with a gluon or collective excitation in the medium (shown by the dashed line in Fig. 2) some quarks can change the color and get excited. If such a quark finds a partner to form a color-neutral pair in the left wing of the resonance it can emit Cherenkov gluon as allowed by the chromopermittivity (argued above in classical terms). This gluon transforms to the quark-antiquark pair. That is how the color-neutral $\bar{q} q g$-component (or four-quark component) of the resonance can be formed from the initial colored two-quark state. Thus beside the common color-neutral $\bar{q} q$-component in the low-mass branch of the $\rho$-meson peak there appears new $\bar{q} q g$-component (as well as others, possibly). Using the wave-line notation for the Cherenkov gluon shown in Fig. 2 one gets a tetraquark state which contributes to the left-wing cross section.

The distributions of the decay products of the left-wing and right-wing states of asymmetric heavy resonances created in the process of nucleusnucleus collisions can slightly differ. If tetraquarks have additional decay modes compared to dimers, these modes can serve for identification of tetraquarks. Then these special modes should reveal themselves only in the low-mass wing of an asymmetric resonance. It is a distinctive feature of $\bar{q} q g$-Cherenkov states. That is more probable for heavy resonances because the presence of a heavy quark in heavy flavor hadrons provides an additional variety of decay channels with new energy scales. Even though the admixture of new decay channels is small, their search deserves special attention.

Surely, the emission of Cherenkov gluons asks for interaction of " $\rho$-meson quarks" within the medium (gluon, collective modes...?). Such an interaction can result either in continuum or quasistable states. In our case, the nature of the primary interaction with $\rho$-meson quarks initiating emission of Cherenkov gluons is however left unknown yet. The main conclusion is that some nuclear forces initiate sometimes the additional binding to tetraquarks by emitted Cherenkov gluons of two otherwise independent unbound color-octet quarks, 


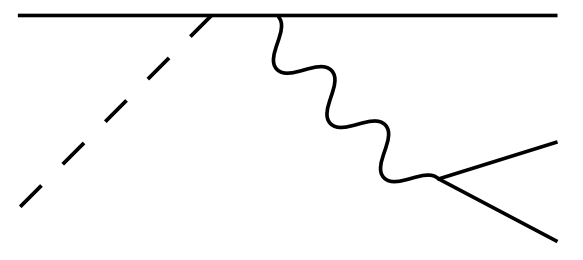

Figure 2: Origin of the tetraquark state. One of the quarks of the initially colored pair interacts within the medium (shown by the dashed line attached to it), changes its color and gets excited, finds its partner for the color-free bound state within the low-mass wing of a resonance, gets de-excited by emission of Cherenkov gluon that leads to the tetraquark states in this wing.

created in the nuclear medium.

The formation of the weakly bound triple-states $(\bar{q} q g)$ in the collisions of three particles when two-particle forces are too weak to produce bound dimers is known as Efimov effect [38, 39. In all the cases the role of the third component is crucial for experimental observation of this effect. It is important to reveal the physics nature of the component. In simplest models, it was ascribed to light quark pairs produced in vacuum in case of narrow resonances [9, 10] and to Cherenkov gluons for wide resonances [35]. 


\section{Conclusion}

To conclude, we have shown that asymmetry of vector mesons produced in nuclear collisions can be successfully described as Fano-Feshbach effect and further interpreted in terms of emission of Cherenkov gluons as a particular detalization of the quantum interference pattern.

From the theoretical side, models of (collective?) excitations in the nuclear medium which help to get an insight to this problem are welcome.

From the experimental side, the error bars in experiments with $\rho$-mesons are quite large. There are some plans to improve experimental accuracy up to two orders of magnitude (private communication). Very little is still known about other resonances but the low-mass asymmetry seems universal and gives some hope for further progress.

The dilepton spectra and, especially, the asymmetry of vector mesons produced in nuclei collisions deserve further precise experimental studies at RHIC and LHC (see discussion in [40]).

\section{Acknowledgments}

We are grateful for support by the RFBR-grant 14-02-00099 and the RAS-CERN program.

\section{References}

[1] R.P. Feynman, R.B. Leighton, M. Sands The Feynman lectures on physics, v. 1 (1963), Addison-Wesley Publishing Company, Inc.

[2] G. Breit, E. Wigner, Phys. Rev. 47, 519 (1936).

[3] PDG group, China Phys. C 38, 090513 (2014).

[4] O.K. Rice, J. Chem. Phys. 1, 375 (1933).

[5] U. Fano, Nuovo Cim. 12, 156 (1935).

[6] H. Feshbach, Ann. Phys. (N.Y.) 5, 357 (1958).

[7] U. Fano, Phys. Rev. 124, 1866 (1961).

[8] R.L. Jaffe, Plenary talk at Hadron 2015 conference. 
[9] R. Shyam, H. Lenske, Phys. Rev. D 90, 014017 (2014).

[10] Xu Cao, H. Lenske, arXiv:1408.5600.

[11] G. Agakichiev et al. (CERES) Phys. Rev. Lett. 75, 1272 (1995); Phys. Lett. B 422, 405 (1998); Eur. Phys. J. C 41, 475 (2005).

[12] D. Adamova et al. (CERES) Phys. Rev. Lett. 91, 042301 (2003); 96, 152301 (2006).

[13] R. Arnaldi et al. (NA60) Phys. Rev. Lett. 96, 162302 (2006).

[14] S. Damjanovic et al. (NA60) Eur. Phys. J. C 49, 235 (2007); Nucl. Phys. A 783, 327 (2007).

[15] D. Trnka et al. Phys. Rev. Lett. 94, 192303 (2005).

[16] M. Naruki et al. (KEK) Phys. Rev. Lett. 96, 092301 (2006).

[17] R. Muto at al. (KEK) Phys. Rev. Lett. 98, 042501 (2007).

[18] A. Kozlov (PHENIX), nucl-ex/0611025.

[19] M. Kotulla (CBELSA/TAPS), nucl-ex/0609012.

[20] I. Tserruya, Nucl. Phys. A 774, 415 (2006).

[21] R. Pisarski, Phys. Lett. B 110, 155 (1982).

[22] M. Harada, K. Yamawaki, Phys. Rep. 381, 1 (2003).

[23] G.E. Brown, M. Rho, Phys. Rev. Lett. 66, 2720 (1991); Phys. Rep. 269, 333 (1996); Phys. Rep. 363, 85 (2002).

[24] K.G. Boreskov, J.H. Koch, L.A. Kondratyuk, M.I. Krivoruchenko, Yad. Fiz. 59, 1908 (1996) [Phys. Atom. Nucl 59, 1844 (1996)].

[25] K. Dusling, D. Teaney, I. Zahed, nucl-th/0604071.

[26] S. Leupold, W. Peters, U. Mosel, Nucl. Phys. A 628, 311 (1998).

[27] J. Ruppert, T. Renk, B. Muller, Phys. Rev. C 73, 034907 (2006). 
[28] M. Chiu, T.K. Hemmick, V. Khachatryan, A. Leonidov, J. Liao, L. McLerran, Nucl. Phys. A 900, 16 (2013).

[29] V.L. Eletsky, M. Belkacem, P.J. Ellis, J.L. Kapusta, Phys. Rev. C 64, 035202 (2001).

[30] V.L. Eletsky, B.L. Ioffe, J.I. Kapusta, Eur. Phys. J. A 3, 381 (1998).

[31] A.T. Martelli, J.P. Ellis, Phys. Rev. C 69, 065206 (2004).

[32] R. Rapp, J. Wambach, Eur. Phys. J. A 6, 415 (1999); Adv. Nucl. Phys. 25, 1 (2000).

[33] H. van Hees, R. Rapp, Phys. Rev. Lett. 97, 102301 (2006).

[34] R.S. Hayano, T. Hatsuda, Rev. Mod. Phys. 82, 2049 (2010).

[35] I.M. Dremin, V.A. Nechitailo, Int. J. Mod. Phys. A 24, 1221 (2009).

[36] A.I. Akhiezer, I.Ya. Pomeranchuk, Nekotorye voprosy teorii yadra (Some problems of theory of nuclei) GITTL, Moscow, 1950, 2nd ed. (in Russian).

[37] I.M. Dremin, A.V. Leonidov, Physics-Uspekhi 53, 1123 (2010).

[38] V. Efimov, Phys. Lett. B 33, 663 (1970).

[39] F. Ferlaino, R. Grimm, Physics 3, 9 (2010).

[40] R. Rapp, Adv. in High Energy Phys. 2013, 148253 (2013). 